\title{
Basin-Wavelength Equatorial Deep Jet Signals across Three Oceans*
}

\author{
MADELEINE K. YounGS \\ NOAA/Pacific Marine Environmental Laboratory, Seattle, Washington, and Environmental Science \\ and Engineering, California Institute of Technology, Pasadena, California \\ GREGORY C. JOHNSON \\ NOAA/Pacific Marine Environmental Laboratory, Seattle, Washington
}

(Manuscript received 3 September 2014, in final form 26 May 2015)

\begin{abstract}
Equatorial deep jets (EDJs) are equatorially trapped, stacked, zonal currents that reverse direction every few hundred meters in depth throughout much of the water column. This study evaluates their structure observationally in all three oceans using new high-vertical-resolution Argo float conductivity-temperaturedepth (CTD) instrument profiles from 2010 to 2014 augmented with historical shipboard CTD data from 1972 to 2014 and lower-vertical-resolution Argo float profiles from 2007 to 2014. The vertical strain of density is calculated from the profiles and analyzed in a stretched vertical coordinate system determined from the mean vertical density structure. The power spectra of vertical strain in each basin are analyzed using wavelet decomposition. In the Indian and Pacific Oceans, there are two distinct peaks in the power spectra, one Kelvin wave-like and the other entirely consistent with the dispersion relation of a linear, first meridional mode, equatorial Rossby wave. In the Atlantic Ocean, the first meridional mode Rossby wave signature is very strong and dominates. In all three ocean basins, Rossby wave-like signatures are coherent across the basin width and appear to have wavelengths the scale of the basin width, with periods of about $5 \mathrm{yr}$ in the Indian and Atlantic Oceans and about $12 \mathrm{yr}$ in the Pacific Ocean. Their observed meridional scales are about 1.5 times the linear theoretical values. Their phase propagation is downward with time, implying upward energy propagation if linear wave dynamics hold.
\end{abstract}

\section{Introduction}

Equatorial deep jets (EDJs) are equatorially trapped, stacked, zonal currents that alternate direction every few hundred meters in depth. The jets are present in all three ocean basins, throughout much of the water column (Johnson et al. 2002; Johnson and Zhang 2003; Luyten and Swallow 1976). In recent years, the effects of the EDJs on water-mass property distributions have been studied in the Atlantic Ocean. For example, Schmid et al. (2005) find that EDJs affect zonal transport of intermediate and deep water masses in the Atlantic.

\footnotetext{
* Pacific Marine Environmental Laboratory Contribution Number 4218.

Corresponding author address: Gregory C. Johnson, NOAA/ Pacific Marine Environmental Laboratory, 7600 Sand Point Way NE, Bldg. 3, Seattle, WA 98115.

E-mail: gregory.c.johnson@noaa.gov
}

Brandt et al. (2011, 2012) argue that as well as ventilating the deep equatorial Atlantic Ocean, the EDJs even force interannual atmospheric variability through their upward energy propagation. We are not aware of similar studies in the Indian and Pacific Oceans, even though the equatorial Pacific strongly affects global climate on interannual and perhaps longer time scales (e.g., Ropelewski and Jones 1987; England et al. 2014). Thus, we are motivated to study the structure and dynamics of the EDJs across all three oceans.

Many different surveys and analyses have focused on EDJs signatures at the equator in the Pacific. In meridional velocity transects at $168^{\circ}$ and $179^{\circ} \mathrm{E}$, dropsonde profiles recorded features with vertical scales of hundreds of meters and time scales longer than the 1-month cruise (Eriksen 1981). These data exhibit very little zonal coherence, perhaps owing to the Gilbert Islands separating the two transects. Using an equatorial transect of dropsonde profiles, Leetmaa and Spain (1981) find zonal currents of $\sim 300$-m vertical scale with a zonal 
coherence of greater than $10^{\circ}$ of longitude. Firing (1987) uses 16 months of dropsonde measurements in meridional transects at $159^{\circ} \mathrm{W}$, collected during the Line Islands Profiling Project as part of the Pacific Equatorial Ocean Dynamics (PEQUOD) campaign, to investigate these deep zonal currents. That study finds EDJs with a vertical scale of $150-400 \mathrm{~m}$ between the thermocline and $3000-m$ depth that exhibit sporadic vertical shifts in position. Ponte and Luyten (1989) perform spectral analysis on 16 months of dropsonde and CTD data collected over $20^{\circ}$ longitude also as part of the PEQUOD campaign to study the EDJs. They see two peaks at 560 and 400-331 stretched meters ( $\mathrm{sm}$ ) and call the latter the EDJs but have a difficult time characterizing the EDJ signal. Using historical CTD data, Johnson et al. (2002) find EDJs in the eastern equatorial Pacific with a vertical wavelength of 400 stretched decibars (sdbar) and a decades-long period.

The use of unstretched and stretched vertical coordinates in various studies makes an exact comparison of wavelengths difficult, as the vertical density profiles used for the stretching would be required. However, the reported EDJ vertical wavelengths appear to be in general agreement. As long Rossby and Kelvin waves are geostrophic, their signatures in vertical strain and zonal velocity should have identical vertical wavelengths (e.g., Eriksen 1982).

Dropsonde measurements in the western Indian Ocean collected in April and June 1979 allow identification of zonal jets of 500-429-sm vertical wavelength at the equator (Ponte and Luyten 1990), longer than that in the Pacific. Velocity and CTD profiles along $80.5^{\circ} \mathrm{E}$ in the Indian Ocean between December 1990 and September 1994 find EDJs with a vertical wavelength of 660 sm (Dengler and Quadfasel 2002).

In the Atlantic Ocean, velocity profiles from sections along $35^{\circ}$ and $13^{\circ} \mathrm{W}$ find EDJs with a vertical scale of $400-600 \mathrm{~m}$, also larger than the vertical scale in the Pacific (Gouriou et al. 1999). Velocity profiles along $35^{\circ}$, $23^{\circ}$, and $10^{\circ} \mathrm{W}$ from the summer of 1999 show coherence of EDJs over $25^{\circ}$ of longitude (Gouriou et al. 2001). Vertical strain sections from historical CTD data show a peak around 661 sdbar in the Atlantic Ocean with a period of $5 \pm 1 \mathrm{yr}$, downward phase propagation, and a zonal wavelength of $70^{\circ} \pm 60^{\circ}$ longitude (Johnson and Zhang 2003). Similar period results are found from Argo float velocity data and much higher temporal resolution moored velocity profiler data (Brandt et al. 2011).

Many studies have interpreted EDJ observations in the Pacific within the framework of linear wave theory. Eriksen (1981) recognizes the need for long-period Rossby waves to explain the width of the jets, but also finds that Kelvin waves may play a role and that short-period, mixed Rossby-gravity waves may help to explain meridional velocities at the equator. Leetmaa and Spain (1981) suggest that the EDJs are either long Rossby or Kelvin waves. The two spectral peaks seen by Ponte and Luyten (1989) are interpreted separately. The peak at $560 \mathrm{sm}$ is characterized as a first meridional mode equatorial Rossby wave, and the peak at 331$400 \mathrm{sm}$ is characterized as a packet of Kelvin waves. Muench et al. (1994) find that the EDJs perturb potential vorticity, a feature that is seen in equatorial Rossby but not Kelvin waves. Johnson et al. (2002) suggest that the EDJs in the eastern Pacific may be consistent with Kelvin wave phase relations but without the benefit of much off-equatorial data to distinguish between Kelvin and Rossby waves. Thus, the interpretation of the EDJs in the Pacific appears ambiguous.

In the Indian Ocean, Ponte and Luyten (1990) find the component of the EDJs with vertical wavelength of $429 \mathrm{sm}$ to be consistent with Kelvin wave phase relations but could not resolve the feature with 500 -sm vertical wavelength. On the other hand, Dengler and Quadfasel (2002) find the EDJs at 660-sm vertical wavelength to be consistent with a nondispersive, first meridional mode Rossby wave by phase relations and meridional distributions of zonal velocity. From current meter moorings in the eastern Atlantic, Weisberg and Horigan (1981) find EDJs to be similar to long Rossby waves. Dropsonde measurements taken at $36^{\circ} \mathrm{W}$ are most consistent with Kelvin wave dynamics (Eriksen 1982). The meridional structure of vertical strain of the EDJs is consistent with first meridional mode Rossby waves, although too broad for simple inviscid theory (Johnson and Zhang 2003). Brandt et al. (2011) find the maximum explained variance of the 1000-m Argo velocities for a high vertical mode, westward-propagating Rossby wave signature of basin wavelength.

This study investigates the EDJs in all three ocean basins to compare and contrast their features. We use vertical strain $\xi_{z}$, a measurement of the squashing and stretching of the density field, to analyze the EDJs. We compute vertical strain from a large quantity of historical shipboard CTD and Argo float profiles. Vertical displacement has also been used to analyze the density field (e.g., Eriksen 1982) but can be aliased by profileto-profile differences in salinity calibrations (Eriksen 1981). To avoid these errors, which could be a very significant source of noise when using data from many different instruments and cruises, we use $\xi_{z}$ instead. We use the Wentzel-Kramers-Brillouin-Jeffreys (WKBJ)scaled stretched pressure (sdbar) as a vertical coordinate to account for the impacts of varying stratification (Leaman and Sanford 1975). We discuss the data used and their processing in section 2 . We provide a qualitative 
description of vertical strain sections from the Pacific in section 3 and follow with quantitative analysis using wavelet decomposition in all three ocean basins in section 4. In section 5, we summarize and discuss the results.

\section{Data and processing}

Here, we use a mix of high-vertical-resolution shipboard CTD and recent Argo profiles, supplemented where necessary by lower-vertical-resolution Argo profiles. Traditionally, owing to a slow data telemetry system and power limitations, Argo floats sample at varying resolutions with a median of around 70 samples per 2000-m profile. Vertical sample spacing for these floats generally increases with increasing depth. Starting in 2006, Argo floats that report data at a vertical resolution of $2 \mathrm{dbar}$ began to be incorporated into the global network, with many of these floats deployed in the equatorial Pacific beginning in 2010. This increased sampling resolution is made possible by the use of the Iridium satellite for communication. Compared to the Argo profiles, the shipboard CTD stations available are quite sparse in space, but owing to their longer historical record, as well as the fact that they sometimes extend to the ocean bottom, they are included in analysis (Fig. 1). Shipboard CTD data were assembled from the National Oceanographic Data Center (NODC), Pacific Marine Environmental Laboratory (PMEL), and CLIVAR and Carbon Hydrographic Data Office (CCHDO) databases.

We find 7113 Argo profiles within $\pm 8.5^{\circ}$ latitude of the equator across the Pacific Ocean dating from January 2010 to May 2014 that reach at least 1990-dbar pressure with no data gaps larger than 20 dbar (Fig. 1; Table 1). These profiles are only from Argo floats using Iridium telecommunications. We add to those data profiles from 2863 shipboard CTD stations reaching at least $1990 \mathrm{dbar}$ and containing no data gaps of greater than $20 \mathrm{dbar}$ within $\pm 8.5^{\circ}$ of the equator across the Pacific (Fig. 1) for the years 1974 to 2012, after carefully screening for and eliminating any possible duplicate stations. Data are sparse from 1972 to 1984, so these years are not plotted in Fig. 1.

There are as of yet very few Iridium Argo floats in the equatorial Indian Ocean, so we use 1965 profiles from traditional and Iridium Argo floats collected from 2007 to 2014 to supplement the 1143 shipboard CTD stations that reach at least 1990-dbar pressure collected from 1978 to 2007 within $\pm 8.5^{\circ}$ latitude of the equator (Fig. 1; Table 1). We only use Argo profiles with what we deem sufficient vertical resolution. In concordance with the traditional Argo float data sampling schemes, and our stretched vertical coordinate system, we require finer vertical sampling closer to the surface. We allow no gaps $>100$ sdbar anywhere in the 400-1990-dbar range over which spectral analyses are applied to these profiles.

Again, there are very few Iridium Argo floats in the equatorial Atlantic Ocean, but because the Atlantic signal has the largest vertical wavelength, and because there are sufficient shipboard CTD profiles to resolve the EDJ signals in the Atlantic (Johnson and Zhang 2003), we use only those 1312 shipboard CTD profiles from the Atlantic reaching at least 3000 dbar collected from 1972 to 2012 within $\pm 8.5^{\circ}$ latitude of the equator and containing no data gaps of greater than $20 \mathrm{dbar}$ (Fig. 1; Table 1).

Profile processing follows Johnson and Zhang (2003). First, the individual profiles are interpolated to a regular 2-dbar pressure grid, whereupon they are low-pass filtered with a 20-dbar half-width Hanning filter and subsampled at 10-dbar intervals (Johnson et al. 2002). The profiles are then used to estimate buoyancy frequency squared $N^{2}=-(g / \rho)(\partial \rho / \partial z)$ by centered differences over 20-dbar spans, where $g$ is the acceleration due to gravity, $z$ is depth, and $\rho$ is the potential density referenced to a local central pressure. Linear wave theory dictates that the local vertical stratification affects the amplitudes and wavelengths of features present in the water column; so, to compensate for this depthdependent factor, WKBJ scaling and stretching is used (Leaman and Sanford 1975). This method compensates for vertical variations in the time-averaged vertical stratification by stretching the vertical coordinate system and scaling the signal amplitudes. Thus, variations of vertical wavelength and amplitude of wave signatures modulated by vertical variations in the time-averaged vertical stratification are minimized, the better to identify features using standard spectral methods.

We compute approximate temporally and laterally averaged vertical profiles of $N$ and $N^{2}$ for each basin, denoted respectively by $\langle N\rangle$ and $\left\langle N^{2}\right\rangle$ as required by the WKBJ scaling (Fig. 2). These quantities are computed by averaging $N$ and $N^{2}$ at each pressure level for every profile within each basin, after which they are smoothed vertically by a 39-point (200-dbar half-width) Hanning filter (Johnson and Zhang 2003). From the filtered profiles, we compute the WKBJ-scaled pressure $p^{*}=\left(1 / N_{o}\right) \int_{0}^{p}\langle N\rangle d p$ for each basin where $N_{o}$ is the pressure-averaged value of $\langle N\rangle$ (Table 2) within that basin. This transformation results in the pressure range for each basin being identical in the stretched and unstretched coordinate systems. We choose the maximum depth of this range to be the zonally averaged depth of each basin along the equator to allow estimates of the 

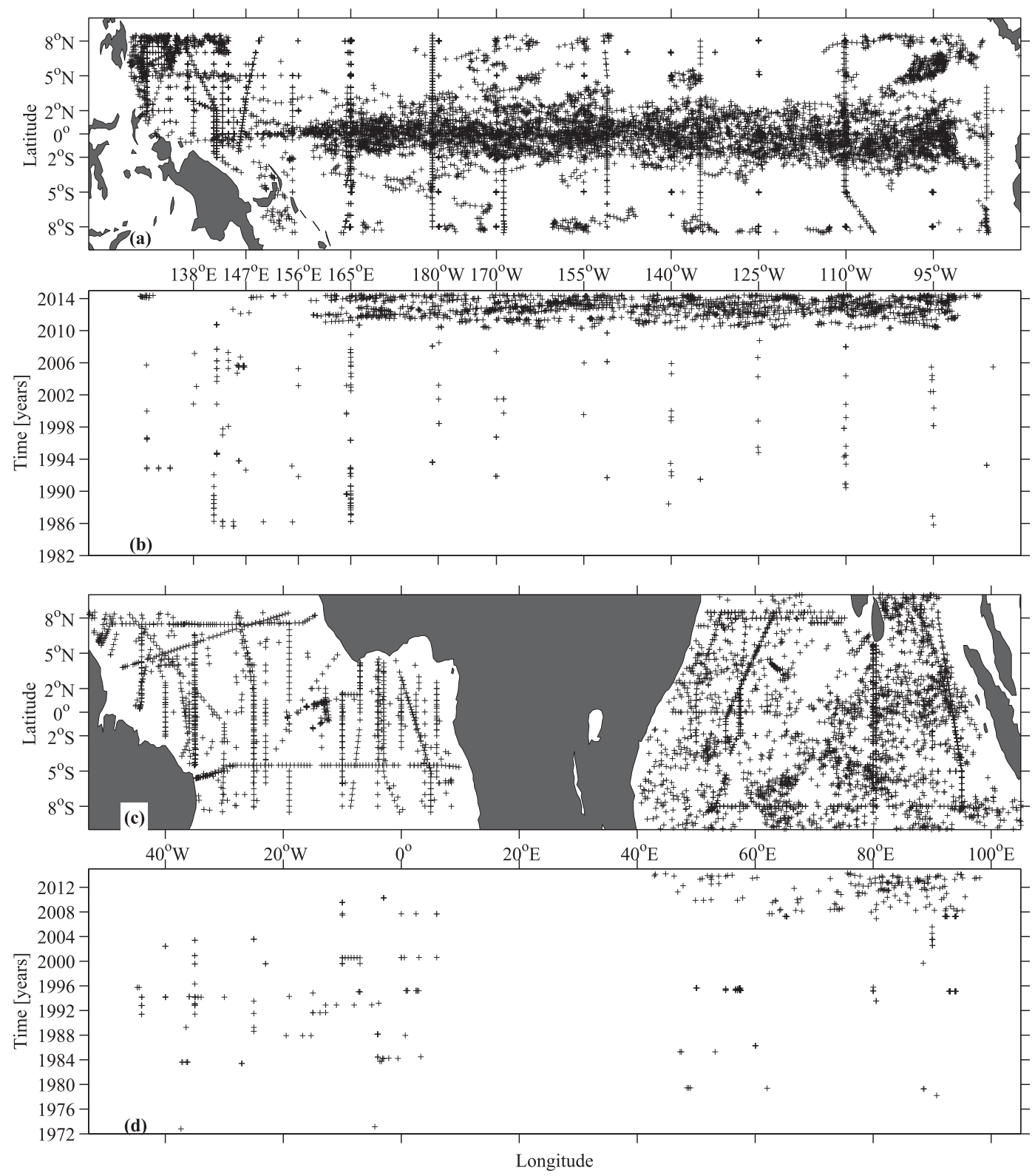

FIG. 1. Geographic locations of deep profiles taken since 1972 in the (a) Pacific and (c) Atlantic and Indian Oceans as well as the longitude-time locations of deep profiles taken within $\pm 0.5^{\circ}$ latitude of $\pm 1.5^{\circ}$ in the (b) Pacific and (d) Atlantic and Indian Oceans.

vertical mode numbers of the EDJs. However, these average depths (Table 2) may be less than those used in other studies, so vertical mode numbers are not necessarily directly comparable.

From $N^{2}$ we compute vertical strain $\xi_{z}=$ $\left(N^{2}-\left\langle N^{2}\right\rangle\right) /\left(\left\langle N^{2}\right\rangle\right)$ to reveal stretching and squashing of the density field. The profiles of $\xi_{z}$ are first estimated on the original pressure grid and then interpolated onto the stretched pressure grid. For this interpolation, if $\langle N\rangle>N_{o}$, then a simple linear interpolation is used, but if $\langle N\rangle<N_{o}$, then the raw values are slightly smoothed to preserve energy for vertical wavelengths of 20 sdbar and longer as a result of simple linear interpolation aliasing short wavelength information (Johnson and Zhang 2003).

We focus on the vertical range from 400 to $1990 \mathrm{dbar}$ in the Indian and Pacific Oceans and 400 to 3000 dbar in the Atlantic, regions where the deep jets are most apparent. While deep jets have been observed as shallow as $250 \mathrm{dbar}$, we limit the top of our range because stratification changes dramatically above 400 dbar and varies widely across basins. Previous studies have noted 
TABLE 1. Summary of profile data used.

\begin{tabular}{lccc}
\hline & Pacific & Indian & Atlantic \\
\hline CTD & 2863 & 1143 & 1312 \\
Argo & - & 1941 & - \\
Iridium Argo & 7113 & 24 & - \\
Total & 9976 & 3108 & 1312 \\
\hline
\end{tabular}

EDJs as deep as 3000 dbar in the Pacific and Indian Oceans (e.g., Johnson et al. 2002; Dengler and Quadfasel 2002), but our range in these two oceans is limited by that of the Argo float data, which are necessary to characterize the EDJs across the entire basins using vertical strain.

\section{Qualitative description}

Since the EDJs have been shown to be equatorially trapped and geostrophic (Eriksen 1982; Muench et al. 1994), their zonal velocity anomalies correspond to squashing and stretching of the density fields at the equator, as exemplified by vertical strain $\xi_{z}$. The relationship between $\xi_{z}$ and zonal velocity depends on the type of wave (Fig. 3). An equatorial Kelvin wave has an on-equatorial maximum amplitude of vertical strain and velocity (Fig. 3a), but a first meridional mode Rossby wave has an off-equatorial maximum in the vertical strain amplitude and an on-equatorial maximum velocity amplitude (Fig. 3b). The maximum amplitude of $\xi_{z}$ is at the equator for Kelvin waves and off the equator for first meridional mode Rossby waves (Fig. 3), making it possible to use $\xi_{z}$ to differentiate the two. In contrast, zonal velocity maxima are at the equator for both waves. The main advantage of $\xi_{z}$ is that density is measured much more often than velocity, allowing basinwide analyses of $\xi_{z}$ over long time scales. Of course, there are other phenomena, such as the much shorter time-scale mixed Rossby-gravity waves, that have a signature in vertical strain fields. Those phenomena are not resolved by the CTD profiles analyzed here and thus are treated as noise.

We discuss vertical strain contoured against pressure versus latitude, longitude, and time in the Pacific to identify the EDJs. The $\xi_{z}$ profiles are smoothed by a loess filter with a half-width of 150 sdbar here, so as to reduce noise while not overly reducing the power spectral peak at the $\sim 250$-sdbar vertical wavelength that will be seen in the wavelet analysis of unsmoothed profiles in section 4 . The profiles used for the meridional and zonal sections were taken between July 2013 and May 2014 to capture waves at one instance in phase of the EDJs, while averaging over the noise of high vertical mode, mixed Rossby-gravity waves and other
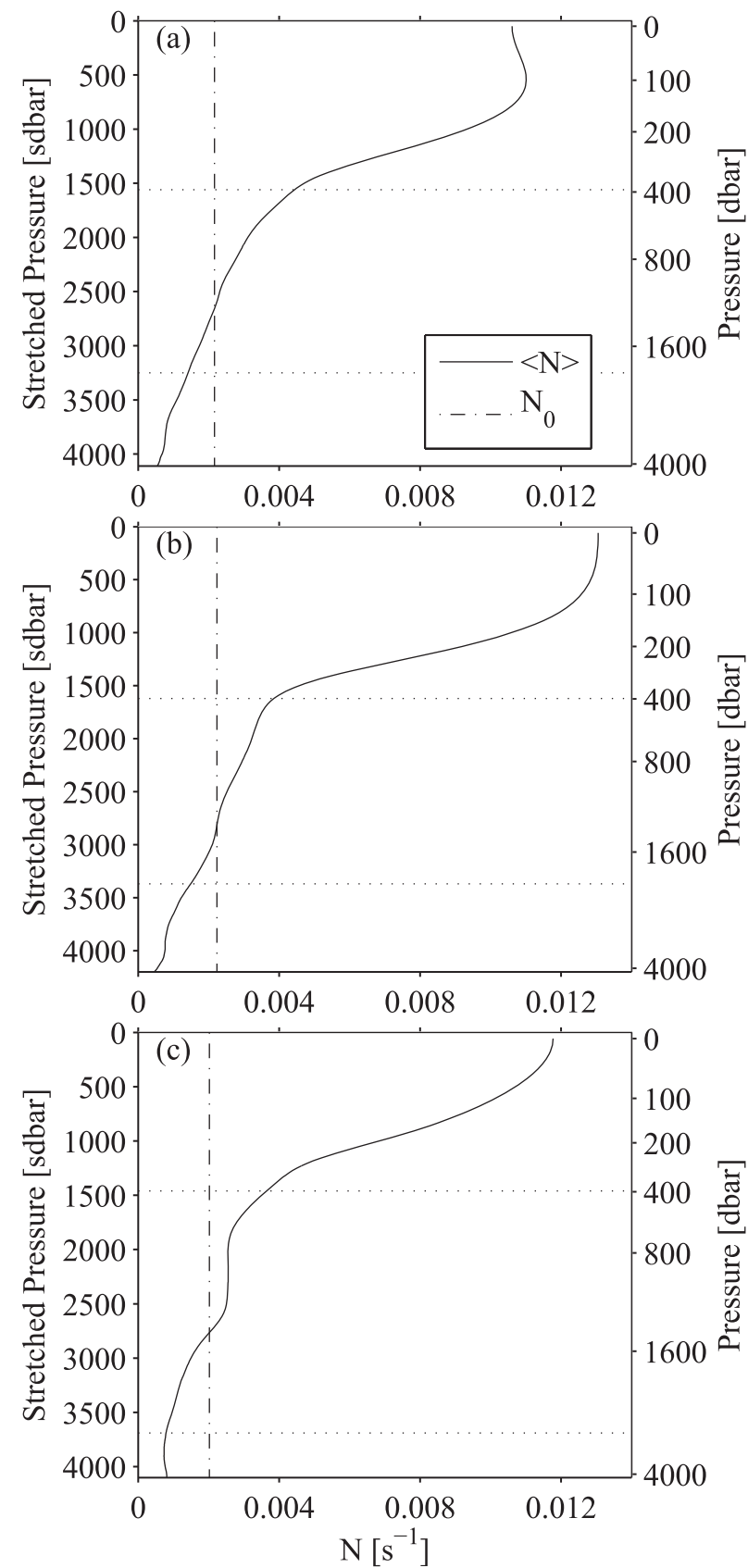

FIG. 2. Profiles of $\langle N\rangle\left(\mathrm{s}^{-1}\right)$ (solid line) plotted against stretched pressure (sdbar) on the left with reference pressures (dbars) on the right for the (a) Pacific, (b) Indian, and (c) Atlantic Oceans. Vertically averaged values $\left(N_{o}\right)$ for each basin are also indicated (vertical dashed lines). Horizontal dotted lines bracket the vertical ranges of the vertical strain profiles used for wavelet analysis in each basin.

high-frequency phenomena. Annual Rossby waves, while prominent, have vertical wavelengths of a few $1000 \mathrm{~m}$ (e.g., Kessler and McCreary 1993), so they are not present in the range of vertical strain vertical wavelengths analyzed here. The time period used limits 
TABLE 2. Quantitative analysis parameters for and characteristics of first meridional mode Rossby waves in all three basins. Parameters include the depth-averaged buoyancy frequency $N_{o}\left(\mathrm{~s}^{-1}\right)$; the values of variance of strain $\sigma^{2}$ used for normalization; the mean bottom depth along the equator; the observed vertical wavelength $\lambda_{z}$; the implied vertical mode given $\lambda_{z}$ and the mean bottom depth; the theoretical ( $l$-theoretical) and observationally estimated ( $l$-fit) meridional scales; the theoretical $\left(\lambda_{x}\right.$-theoretical) and observationally estimated $\left(\lambda_{x}\right.$-fit) zonal wavelengths; and the observationally estimated periods of the waves.

\begin{tabular}{|c|c|c|c|}
\hline & Pacific & Indian & Atlantic \\
\hline$N_{o}\left(\mathrm{~s}^{-1}\right)$ & 0.0022 & 0.0022 & 0.0020 \\
\hline Mean variance $\left(\sigma^{2}\right)$ & 0.0885 & 0.0625 & 0.0987 \\
\hline Mean bottom depth (dbar) & 4050 & 4200 & 4100 \\
\hline$\lambda_{z}(\mathrm{sdbar})$ & $207<360<933$ & $203<428<961$ & $373<467.5<635$ \\
\hline Vertical mode & $39>22>8$ & $41>19>8$ & $21>17>12$ \\
\hline$l$-theoretical & $0.51^{\circ}<0.67^{\circ}<1.08^{\circ}$ & $0.51^{\circ}<0.73^{\circ}<1.09^{\circ}$ & $0.65^{\circ}<0.73^{\circ}<0.85^{\circ}$ \\
\hline$l$-fit & $1.00^{\circ}$ & $1.09^{\circ}$ & $1.08^{\circ}$ \\
\hline$\lambda_{x}$-theoretical & $144^{\circ}$ & $71^{\circ}$ & $71^{\circ}$ \\
\hline$\lambda_{x}$-fit & $87^{\circ}<127^{\circ}<234^{\circ}$ & $36^{\circ}<67^{\circ}<445^{\circ}$ & $56^{\circ}<68^{\circ}<89^{\circ}$ \\
\hline Period-fit (years) & $10<12<15$ & $4.4<4.9<5.6$ & $4.6<4.8<5.0$ \\
\hline
\end{tabular}

the data to high-resolution Argo profiles. The contouring is done by ordinary linear interpolation.

Smoothed, meridional-vertical strain at two wellsampled meridians in the western and eastern Pacific Ocean (Fig. 4) displays signatures of the EDJs. At both $165^{\circ} \mathrm{E}$ and $110^{\circ} \mathrm{W}$, an off-equatorial maximum is seen at around $\pm 1^{\circ}$ to $\pm 1.5^{\circ}$ latitude in the sections. This offequatorial maximum is stronger and perhaps farther from the equator at $165^{\circ} \mathrm{E}$. The off-equatorial signal is also more coherent deeper in the water column, whereas the on-equatorial signal is more coherent shallower in the water column. There also appears to be a longer vertical wavelength-about $350 \mathrm{sdbar}$-for the feature at $\pm 1.5^{\circ}$ latitude than for the feature at the equator, which has a vertical wavelength of about 250 sdbar.

Smoothed zonal-vertical strain fields within $0.25^{\circ}$ latitude of the equator and of $\pm 1.5^{\circ}$ latitude for July 2013 to May 2014 exhibit coherence over different zonal scales (Fig. 5). Along the equator, the vertical strain is not obviously coherent over large zonal or vertical scales. In contrast, at $\pm 1.5^{\circ}$ latitude, the signal is visually coherent over the pressure range considered and across the entire basin. The zonal wavelength is visually estimated to be the width of the basin, with the phase propagating downward to the east, especially east of the date line.

Smoothed equatorial versus off-equatorial temporalvertical strain fields in the eastern equatorial Pacific (Fig. 6) also have differing characteristics, upon visual analysis. At the equator, visual inspection suggests a signal in vertical strain that may be propagating upward with time with a period of about $2.5 \mathrm{yr}$, but the weak coherence makes that conclusion very speculative. In contrast, at $\pm 1.5^{\circ}$ latitude, the signal seems to propagating downward with time with a period of about $12 \mathrm{yr}$ over the entire pressure range. Of course, inferring a 12 -yr period from visual inspection of a 4-yr record implies a very tentative estimate, but the signal at $\pm 1.5^{\circ}$ latitude is much more coherent than the signal at the equator.

In summary, there are two different latitudes in the Pacific at which there are maxima in the amplitude of the strain fields, one at the equator and the other at around $\pm 1.5^{\circ}$ latitude (Fig. 4). The peak in the strain field at the equator is broadly consistent with the structure of an equatorial Kelvin wave, and the off-equatorial peak in the strain field is broadly consistent with an equatorial first meridional mode Rossby wave, except for some indications of hemispheric asymmetry, which are addressed below. The equatorially peaked feature might be propagating upward in time with a period of $2.5 \mathrm{yr}$ (Fig. 6a) but lacks the coherence to determine the zonal scale. The off-equatorially peaked feature seems to be propagating downward with time with a period of about $12 \mathrm{yr}$ and propagating downward to the east with a zonal wavelength of the width of the basin (Figs. 5b, 6b). Overall, the off-equatorial signal is much more coherent than the on-equatorial signal.

\section{Quantitative analysis}

EDJs in the Pacific have been shown to be localized below the thermocline in the water column with maximum amplitude near $2000 \mathrm{dbar}$ (e.g., Johnson et al. 2002). Because EDJs may vary with pressure, wavelet analysis (Torrence and Compo 1998) is well suited for an energy and phase analysis of the EDJs. The profiles used for wavelet analysis are not the smoothed profiles used in section 3 but instead the interpolated profiles that should resolve signals down to a 40-sdbar vertical wavelength. The coarser sampling of the traditional Argo profiles we use in the Indian Ocean means that some of the shorter vertical wavelength energy will be lost, resolving down to 200 -sdbar vertical wavelength, 

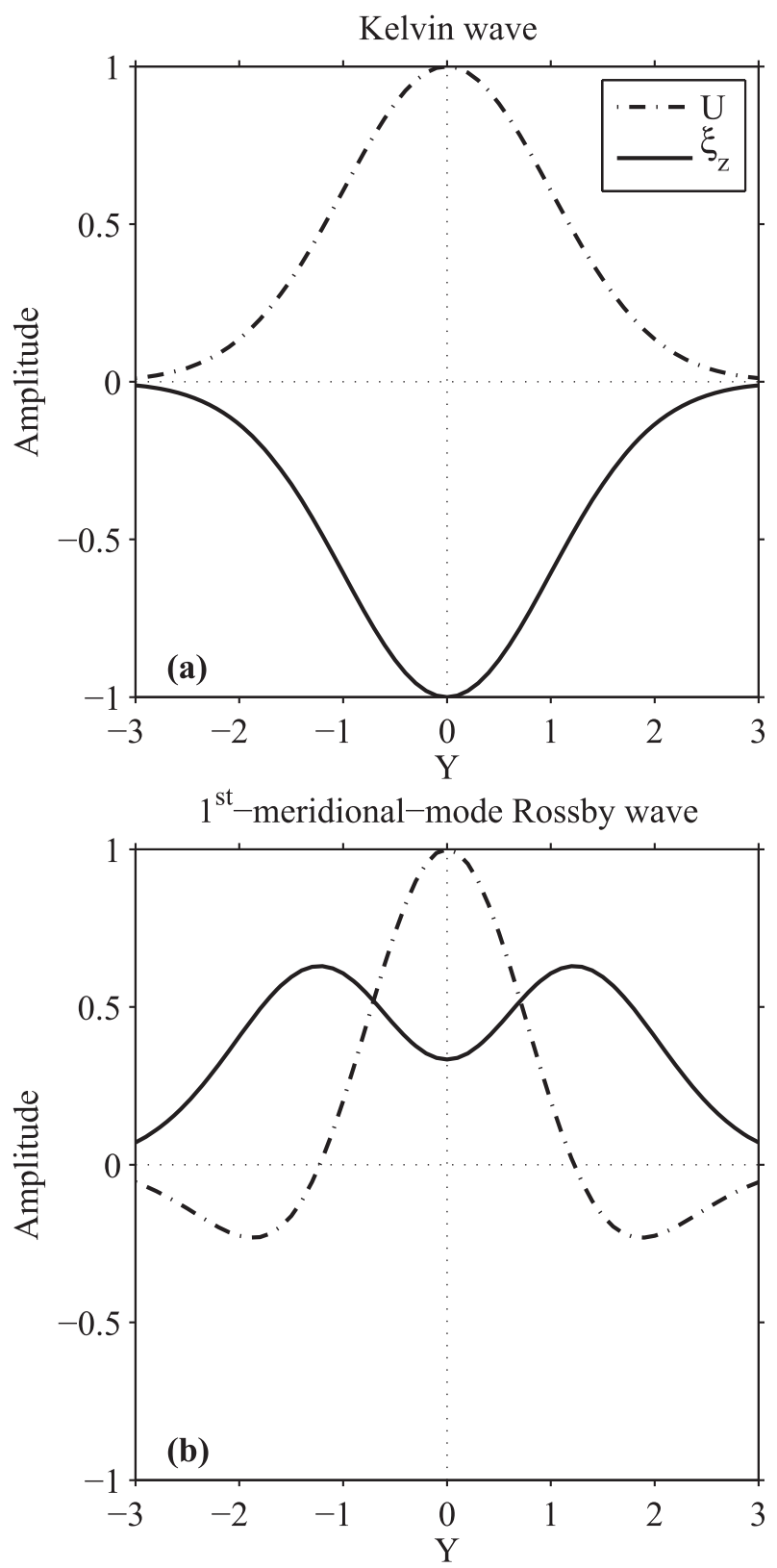

FIG. 3. Schematic of meridional structure of zonal velocity ( $U$, dotted-dashed line) and vertical strain $\left(\xi_{z}\right.$, solid line) for (a) an equatorial Kelvin and (b) a first meridional mode equatorial Rossby wave. Each $x$ axis is normalized by the Kelvin wave meridional scale, and $y$ axes show scaled amplitudes. Figure follows Johnson and Zhang (2003).

but with the restrictions we put on vertical gaps in the data for those profiles, they still well resolve the EDJ signals. We apply wavelet analysis over 400-2000 dbar in the Pacific and Indian Oceans, and 400-3000 dbar in the Atlantic Ocean, because these are regions where $\langle N\rangle$ does not vary too much laterally, so the stretching and scaling is likely to be valid (Eriksen 1981). Since $\xi_{z}$ is a normalized, prewhitened quantity, no preparation is required for the wavelet analysis (Johnson and Zhang 2003). We use a Morlet wavelet as the wavelet function, following Johnson and Zhang (2003). The profiles are zero padded to minimize edge effects, and the regions where edge effects are important are blanked out. The spectra for each basin are normalized by the mean variance $\sigma^{2}$ of all profiles in each basin that are located further than $\pm 3^{\circ}$ latitude from the equator (Table 2). This normalization allows us to look at near-equatorial departures from an off-equatorial background level of vertical strain variance. The reduced vertical resolution of the traditional Argo profiles included in the Indian Ocean analysis may be part of the reason that $\sigma^{2}$ is lower there than in the other two oceans.

Mean power spectra reveal meridional and vertical structure of the EDJs (Fig. 7). We analyze nonoverlapping bins centered at $0^{\circ}, \pm 0.33^{\circ}, \pm 0.67^{\circ}, \pm 1^{\circ}$, $\pm 1.5^{\circ}, \pm 2^{\circ}, \ldots, \pm 5^{\circ}$ latitude. In all three ocean basins, we see peaks in the power spectrum on and off the equator (Fig. 7). In both latitude ranges, peaks are located near the center of the pressure range. We focus our discussions on these peaks.

In all three oceans, the equatorial peak has a slightly shorter vertical wavelength than the off-equatorial peak (Fig. 7). The equatorial and off-equatorial peaks are all strongest in the Atlantic and weakest in the Pacific Ocean. The vertical wavelength of the off-equatorial peak is longest in the Atlantic and shortest in the Pacific (Fig. 7; Table 2). Also, in every ocean basin there is an equatorial peak localized in the upper part of the water column (around $800 \mathrm{dbar}$ ) with a significantly shorter vertical wavelength than the other features. This peak may not be related to the EDJs.

The power spectra along the center of the pressure range analyzed (976 dbar in the Pacific, 1034 dbar in the Indian, and $1240 \mathrm{dbar}$ in the Atlantic) for the bins at various distances from the equator show the meridional structures of the various peaks (Fig. 8). The Pacific Ocean has a very broadband peak at the equator with a vertical wavelength of 120-400 sdbar and a much narrower peak near $\pm 1^{\circ}$ latitude with a vertical wavelength of 360 sdbar (Fig. 8a). The Indian Ocean has similar broadbanded structure near the equator but perhaps bracketed by distinct peaks at 120 and 400 sdbar, again with a narrow peak at 428 sdbar around $\pm 1.5^{\circ}$ latitude (Fig. 8b). In the Atlantic, there is a very strong peak near $\pm 1.5^{\circ}$ latitude at a 467 -sdbar vertical wavelength and a weaker peak near the equator at 400 -sdbar vertical wavelength (Fig. 8c). Here, we suggest that the distribution of power with increasing distance from the equator at the vertical wavelengths of the equatorial spectral peaks is a combination of Kelvin waves, which 

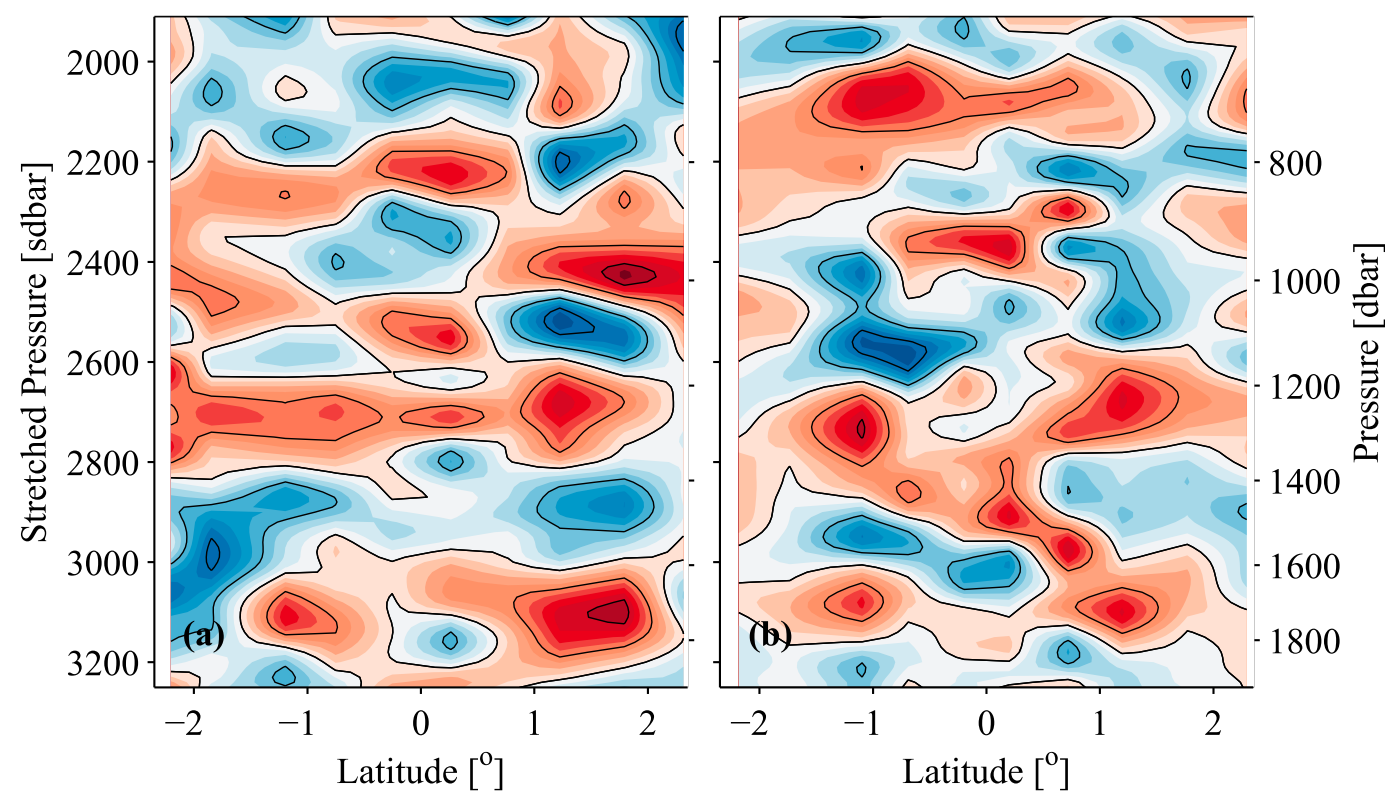

FIG. 4. Meridional section of smoothed $\xi_{z}$ within $\pm 2.5^{\circ}$ longitude of (a) $165^{\circ} \mathrm{E}$ and (b) $110^{\circ} \mathrm{W}$ using high-verticalresolution Argo CTD data taken from July 2013 to May 2014. Strain profiles are averaged in $0.5^{\circ}$ latitude bins prior to contouring. Contour interval is 0.2 for black lines with negative values blue and positive values red.

decay with distance from the equator, and first meridional mode equatorial Rossby waves, which have an offequatorial peak. In what follows, we focus more on the off-equatorial peaks, showing that their vertical wavelength, period, and zonal wavelength are all consistent with the dispersion relation for first meridional mode equatorial Rossby waves in each ocean basin. Furthermore, their meridional structure, while somewhat broader than predicted by theory, otherwise agrees with it as well.

The power of $\xi_{z}$ of a first meridional mode equatorial Rossby wave is given by $b\left\{\left[1+2(y / l)^{2}\right] \exp \left[-0.5(y / l)^{2}\right]\right\}^{2}$. For an equatorial Kelvin wave, power is given by $d\left\{\exp \left[-0.5(y / l)^{2}\right]\right\}^{2}$. Here, $b$ is the Rossby wave energy level and $d$ is the Kelvin wave energy level, and $l=(c / \beta)^{0.5}$ is the meridional scale, with $\beta=2.3 \times$ $10^{-11} \mathrm{~m}^{-1} \mathrm{~s}^{-1}$ being the meridional derivative of the Coriolis parameter and $c=\left(\lambda_{z} N_{o}\right) /(2 \pi)$ being the Kelvin wave phase speed. Using the power spectra at the pressures $(1049 \mathrm{dbar}$ in the Pacific Ocean, $906 \mathrm{dbar}$ in the Indian, and 1240 dbar in the Atlantic) and latitude bins $\left( \pm 1^{\circ}\right.$ in the Indian and Pacific Oceans and $\pm 1.5^{\circ}$ in the Atlantic) with maximum off-equatorial signal in each basin, the vertical wavelengths for which the variance drops to half-maximum from the peak amplitude are used for uncertainty ranges (Table 2). This information is used to compute the likely ranges of $l$ and vertical mode number (for the zonally averaged depth, perhaps less than the depth used in previous studies) in each ocean (Table 2).
We can further quantify the meridional structure of the EDJs by examining the power at the pressure of the maximum variance at the longer vertical wavelength (off equatorial) peak in each basin (Fig. 9) as a function of latitude. We use the same nonoverlapping latitude bins for this purpose, fitting the observed mean meridional structure of power to that predicted for equatorial Kelvin and Rossby waves of energy $d$ and $b$, respectively, along with a background energy level $a$ (Fig. 9). In addition to those three free parameters, we allow $l$, the meridional scale for the waves, to vary from the a priori theoretical value in each basin (Table 2). The observational estimates of $l$ are larger by a factor of 1.5 than the theoretical values in all three basins. However, only the observational estimate of $l$ in the Atlantic Ocean disagrees significantly with the theoretical prediction from linear wave theory when the confidence limits (given by the uncertainties in vertical wavelengths derived from the widths of the spectral peaks) are considered. At the wavelength and pressure levels analyzed in each basin, the fits again suggest that the very strong Rossby wave signature dominates in the Atlantic Ocean, even at the equator. In contrast, in the Indian and Pacific Oceans the Kelvin wave signatures have slightly higher peak energies than the Rossby wave signatures. Overall, equatorial planetary wave energy levels are intermediate in the Indian Ocean and lowest in the Pacific. However, even in the Indian and Pacific Oceans, the Rossby wave signature dominates the vertical strain off the equator. 

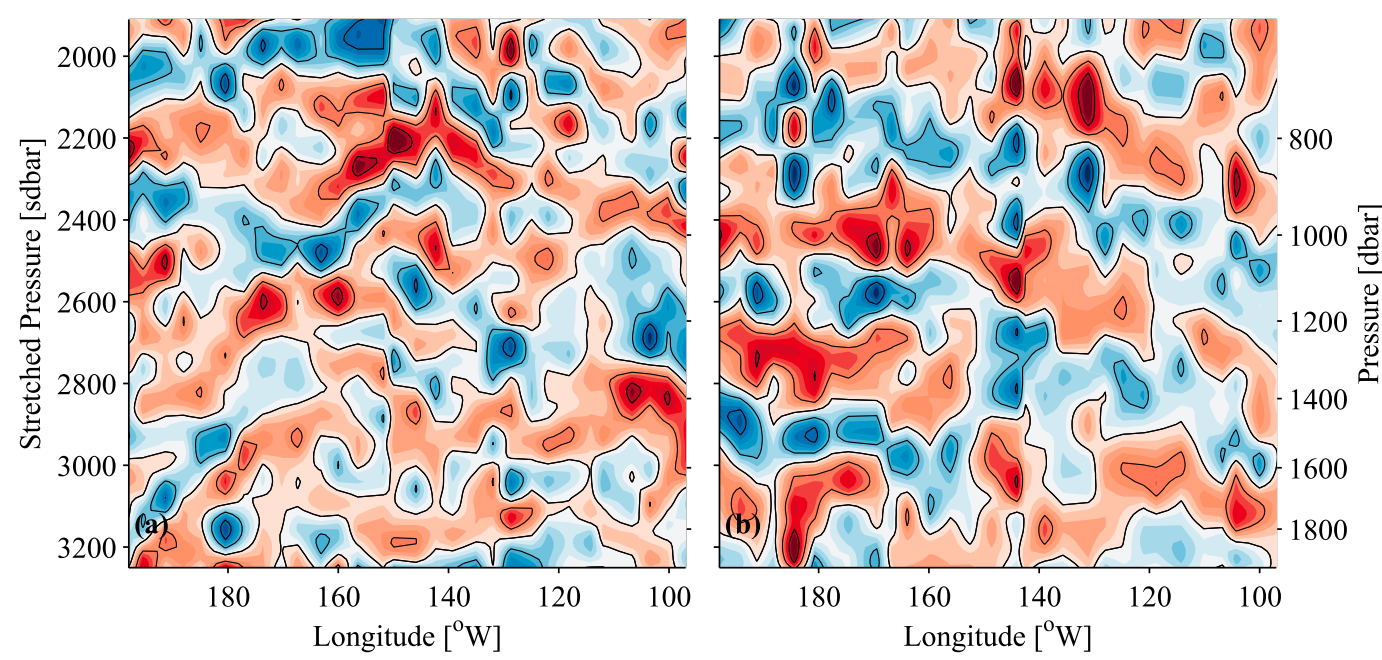

FIG. 5. Zonal section of smoothed $\xi_{z}$ within $\pm 0.25^{\circ}$ latitude of (a) the equator and (b) $\pm 1.5^{\circ}$ latitude. Strain profiles are averaged in $3.5^{\circ}$ longitude bins prior to contouring. Other details are as in Fig. 4.

Finally, in each ocean basin we estimate periods and zonal wavelengths from observations by fitting a plane wave [e.g., $\left.\sin \left(2 \pi x / \lambda_{x}-2 \pi t / \tau+\phi\right)\right]$, where the free parameters are the zonal wavelength $\lambda_{x}$, the period $\tau$, and the phase offset $\phi$ [see Johnson and Zhang (2003) for more information on plane wave fitting]. We make these fits to phase estimates from each profile for the coherent, narrowband, off-equatorial Rossby wavelike peaks in the power spectra (Table 2), again at the pressures where the peaks are a maximum in each basin (Fig. 9). For each basin we carefully select an off-equatorial latitudinal band and a cutoff variance below which we do not attempt to fit the phase estimate from a profile (Fig. 10). The plane waves explain only a fraction of the variance, and results are somewhat sensitive to choices of latitude bands and cutoff variances. Our selections minimize uncertainties in the fits by concentrating on phase estimates from profiles with a strong signal. Nonetheless, there is a significant spread in the phase residuals, especially in the Pacific and Indian Oceans (Fig. 10). In the Atlantic Ocean, the signal variance is much higher, and the fit is better. Again, the plane waves explain only a fraction of the variance in each basin, but they do indicate coherent signatures of the EDJs at basin scales and very long time periods. Furthermore, while there is sometimes asymmetry of the off-equatorial signal in quasi-synoptic sections (Fig. 4), basinwide coherent signals isolated by the plane wave fits are indistinguishable when the analysis presented here is performed separately in each hemisphere (not shown). The coherent signals appear to be symmetric across the equator, as expected for long Rossby waves.
The periods estimated from these plane wave fits are $12(10-15) \mathrm{yr}$ in the Pacific, $4.8(4.6-5.0) \mathrm{yr}$ in the Atlantic, and 4.9 (4.4-5.6) yr in the Indian Ocean (Fig. 10; Table 2). The zonal wavelengths estimated from the fits are $127^{\circ}\left(87^{\circ}-234^{\circ}\right)$ longitude in the Pacific, $68^{\circ}\left(56^{\circ}-89^{\circ}\right)$ in the Atlantic, and $67^{\circ}\left(36^{\circ}-445^{\circ}\right)$ in the Indian Ocean. The $2.5 \%-97.5 \%$ uncertainty ranges in parenthesis are calculated here using effective degrees of freedom estimated following Johnson and Zhang (2003) but correcting an error in how they applied those degrees of freedom in the uncertainty estimates and calculating those uncertainties for frequencies and zonal wavenumbers rather than periods and zonal wavelengths. In all three oceans, phase propagation for these fits is westward and downward in time, with the latter suggesting upward energy propagation if these features are indeed linear, first meridional mode, equatorial Rossby waves. While the uncertainties for the zonal wavelengths are large, their central values are on the order of the zonal width of their respective basins at the equator. Also, given the vertical wavelength and the estimates of the period in each basin, the zonal wavelengths predicted for a first meridional mode equatorial Rossby wave agree very well with the central values of the observational estimates of that quantity from the plane wave fits (Table 2). Here, the theoretical zonal wavelengths are given by the linearized, first meridional mode, Rossby wave dispersion relation $\lambda_{x}=(c / 3) T$, where $c$ is the Kelvin wave phase speed (Table 2). We estimate $c$ from the observational estimate of the vertical wavelength $\lambda_{z}$ from the spectral peak and the observational period $T$ estimated from the planar fit. Increasing variance to the west at those wavelengths, 

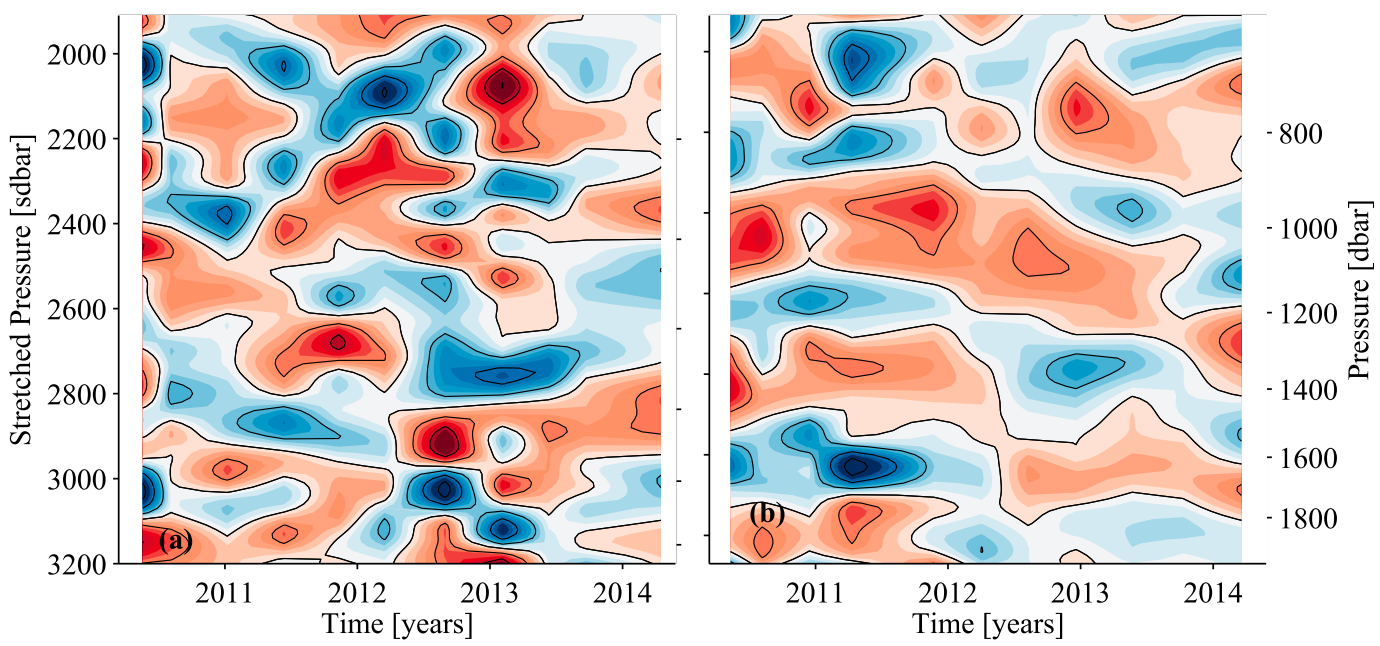

FIG. 6. Temporal section of smoothed $\xi_{z}$ within $\pm 0.6^{\circ}$ latitude of (a) the equator and (b) $\pm 1.5^{\circ}$ latitude within $\pm 5^{\circ}$ longitude of $110^{\circ} \mathrm{W}$ using high-vertical-resolution Argo CTD data taken from 2010 to May 2014. Strain profiles are averaged in 0.4-yr bins prior to contouring. Other details follow Fig. 4.

pressures, and off-equatorial latitudes is also apparent in all three ocean basins (Fig. 10).

\section{Discussion}

Vertical strain signatures in all three ocean basins exhibit a relatively broadband spectral peak at the equator over a large range of pressures below the thermocline with vertical wavelengths of a few hundred stretched decibars (Fig. 7) and variance amplitudes significantly larger than background levels found a few degrees or more from the equator (Fig. 8). The decay in amplitude of these peaks with increasing distance from the equator is consistent with high vertical mode, equatorial Kelvin waves (Fig. 9). However, we are unable to find large-scale zonal or temporal coherence to this signal, perhaps owing to its broadband nature caused by the superposition of Rossby and Kelvin wave signals.

In contrast, there is a narrowband, off-equatorial peak with slightly longer vertical wavelengths than the broadband equatorial peak (Figs. 7, 8) in all three basins. The pattern of variance amplitude for this peak with distance from the equator is grossly consistent with the structure of the first meridional mode, equatorial Rossby wave, although its meridional scale is about 1.5 times wider than the theoretical scale in all three oceans (Fig. 9; Table 2). These Rossby wave-like structures exhibit large-scale zonal (Fig. 5) and longtime temporal (Fig. 6) coherence. Fits of a plane wave to the phase of these off-equatorial peaks in each basin, while somewhat noisy in the Indian and Pacific Oceans (Fig. 10), nonetheless confirm coherent signals across the basin and over the sampling times with observational estimates of vertical wavelengths, periods, and zonal wavelengths that are completely consistent with the dispersion relation for first meridional mode, equatorial Rossby waves (Table 2). In all three ocean basins, the phase propagation for this signal is westward and downward in time, consistent with a Rossby wave and suggesting upward energy propagation if linear wave theory is applicable.

There are large differences in the variance of these signals in the different basins (Figs. 7, 8, 9). The strongest, most coherent signal is the Rossby wave-like one in the Atlantic Ocean, which dominates in that basin. In the Indian and Pacific Oceans, the Rossby wave-like and Kelvin wave-like signals are of similar amplitudes, with the Pacific having the smallest amplitude signals. In addition, variance of the Rossby wave-like signal appears to increase to the west at the pressures of maximum variance and the vertical wavelengths of the off-equatorial spectral peaks in all three ocean basins (Fig. 10). Of course, given the sparse sampling in space and time in the Atlantic and Indian Oceans, and the short (with respect to an estimated $12-\mathrm{yr}$ period) $4 \mathrm{yr}$ of intense sampling in the Pacific (Fig. 1), it is possible that this pattern is aliased. On the other hand, it does appear in all three ocean basins.

Over the years, EDJs have been interpreted differently using linear wave theory, but the different results are not as inconsistent as they first may seem. Ponte and Luyten (1989) find two peaks in their power spectra in the equatorial Pacific Ocean, one at $560 \mathrm{sm}$ and the other at $331-400 \mathrm{sm}$. They characterize the peak at $560 \mathrm{sm}$ as a first meridional mode Rossby wave and the peak 
$\xi_{\mathrm{z}}$ wavelet power spectrum for $|1 \mathrm{at}| \leq 0.17^{\circ} \xi_{\mathrm{z}}$ wavelet power spectrum for $0.83^{\circ} \leq|\mathrm{lat}| \leq 1.25^{\circ}$
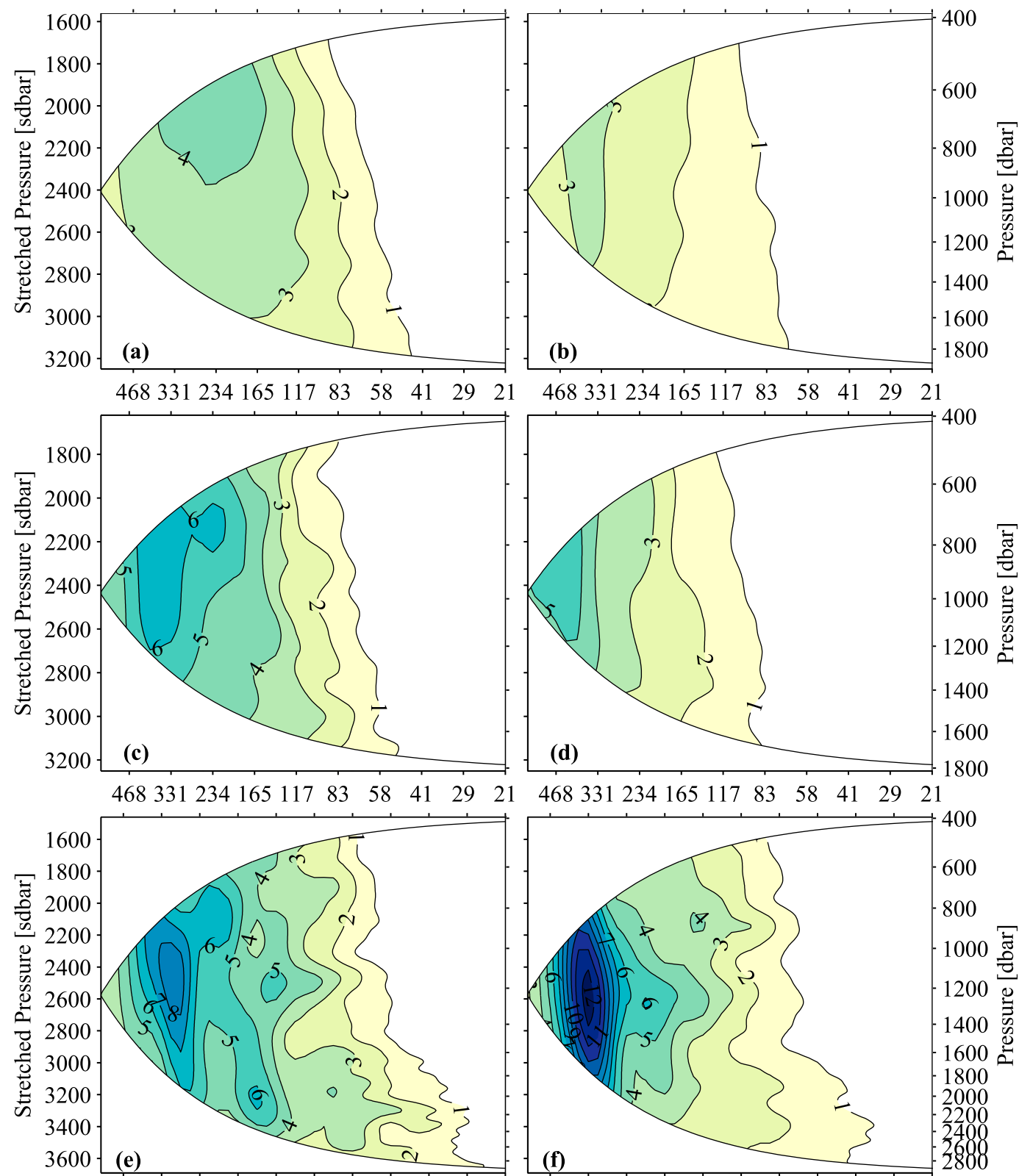

$66146833123416511783 \quad 58 \quad 41 \quad 2921 \quad 66146833123416511783 \quad 58 \quad 41 \quad 2921$

Vertical wavelength [sdbar]

Vertical wavelength [sdbar]

FIG. 7. Power spectra of $\xi_{z}$ from all profiles (left) within $\pm 0.17^{\circ}$ latitude of the equator and (right) between $\pm 0.83^{\circ}$ and $\pm 1.25^{\circ}$ latitude of the equator in the (a),(b) Pacific, (c),(d) Indian, and (e),(f) Atlantic Oceans. Contour intervals for variance are $\sigma^{2}$, where individual profiles have been normalized by the mean variance of profiles reaching $1990 \mathrm{dbar}$ poleward of $3^{\circ}$ latitude from the equator in each ocean basin (Table 2).

at $331-400 \mathrm{sm}$ as a packet of Kelvin waves. If we adjust for different $N_{o}$ used, the wavelengths of the peaks are nearly identical to those we find. Their interpretations of the different features are also almost identical to ours. The main difference is that Ponte and Luyten (1989) do not include the peak at 560 -sm vertical wavelength as a component of the EDJs. From Fig. 4 and the analysis done in section 3, it is clear that a component of the EDJs includes the Rossby wave-like signal. Johnson et al. (2002) interpreted the Pacific EDJs as a Kelvin wave. However, they were only able to find a coherent phase pattern over a range of only $50^{\circ}$ longitude in the 

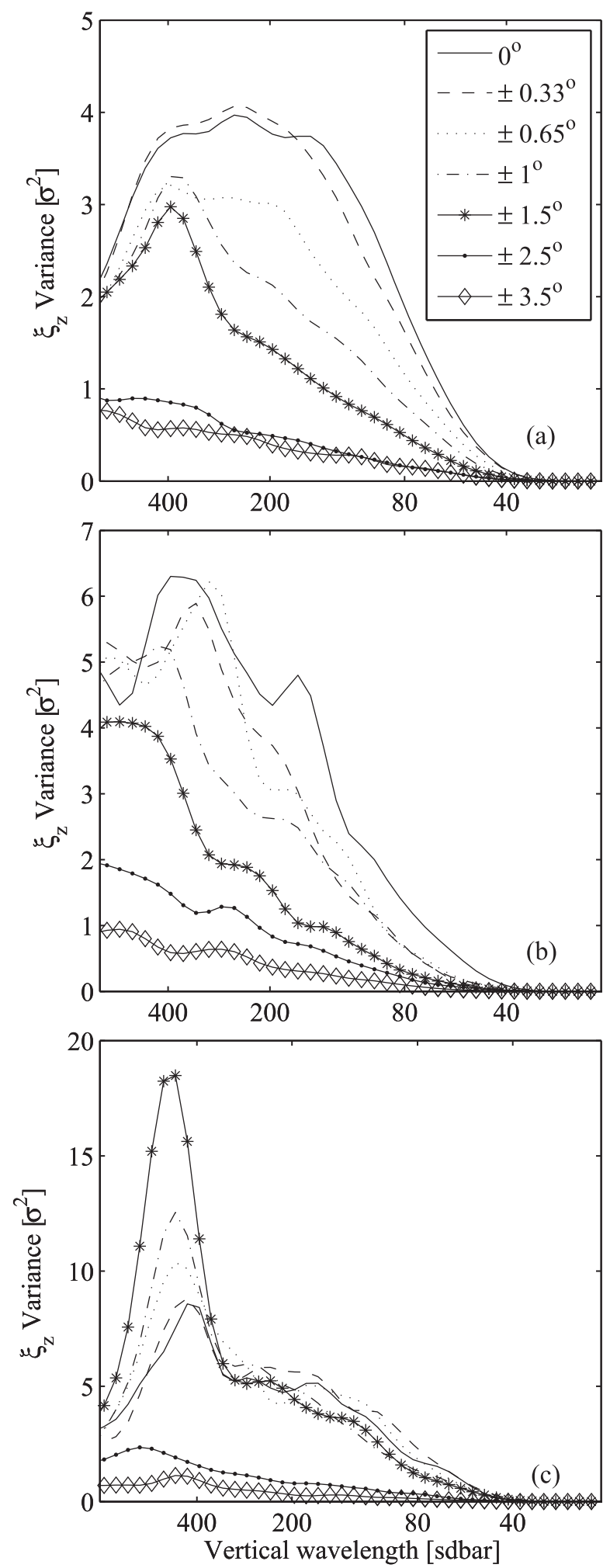

FIG. 8. Average variance squared vs vertical wavelength for various latitude bins (see legend) across the (a) Pacific, (b) Indian, and (c) Atlantic Oceans. The power is taken from a depth of 976 dbar (2410 sdbar) in the Pacific, 1034 dbar (2500 sdbar) in the Indian, and 1240 dbar (2580 sdbar) in the Atlantic Ocean to maximize the vertical wavelengths resolved. Vertical scales vary from smallest in the Pacific to largest in the Atlantic.
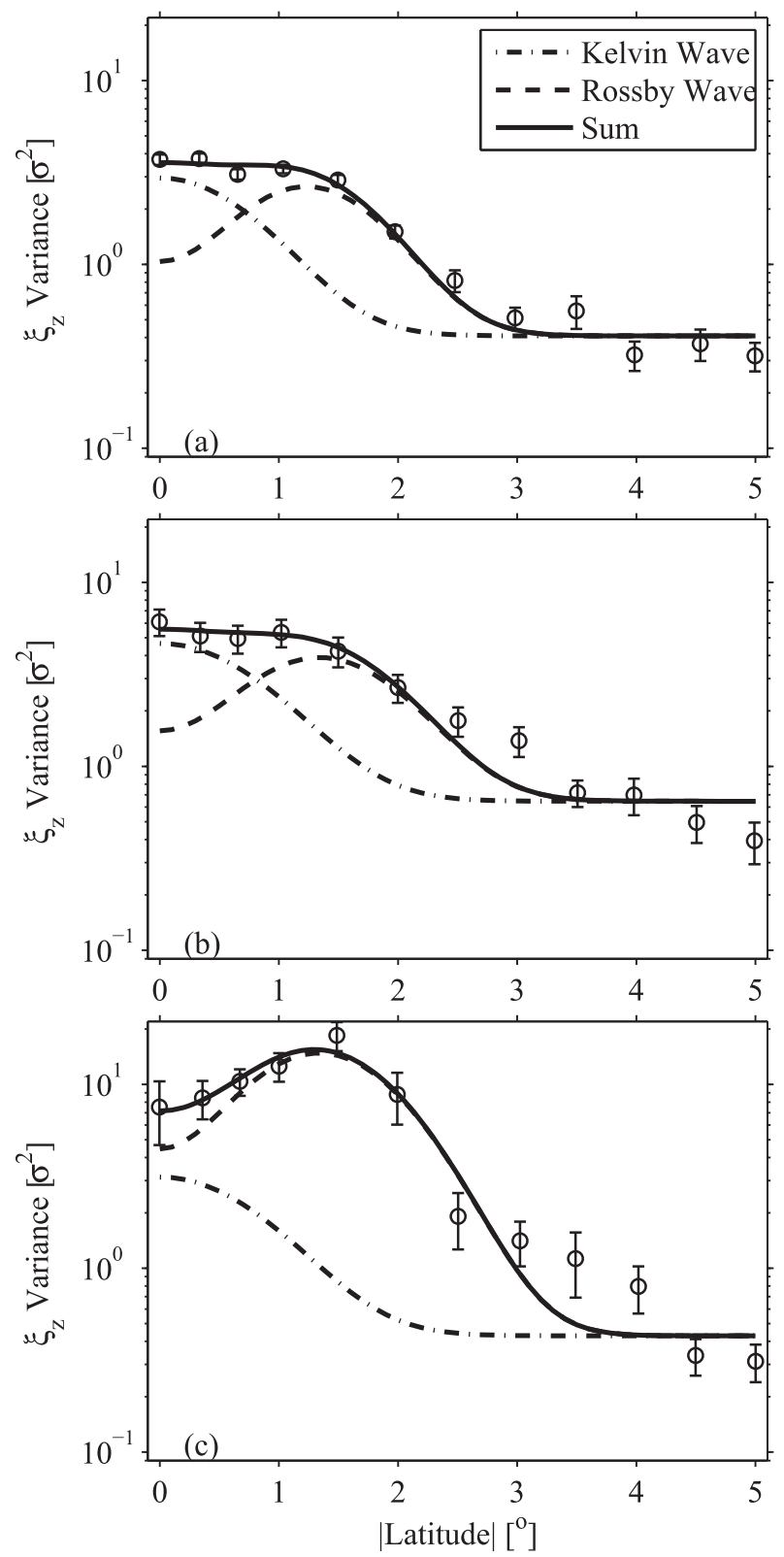

FIG. 9. Average power of vertical strain binned by distance from the equator at the vertical wavelengths and pressures of the peaks around $\pm 1.5^{\circ}$ latitude in Fig. 8 in the (a) Pacific, (b) Indian, and (c) Atlantic Oceans. Two standard errors of the mean (error bars) are used to approximate two-tailed $95 \%$ confidence intervals. Amplitudes of fitted Rossby waves (dashed lines), fitted Kelvin waves (dashed-dotted lines), and the sums of the two (solid lines) are shown for each basin.

eastern Pacific, so their results are limited. They estimated the period to be decades long, a result that is inconsistent with the equatorial Kelvin wave dispersion relation. However, the analyses in Johnson et al. (2002) do reveal a peak in the power spectrum at a vertical wavelength longer than the Kelvin wave feature identified 

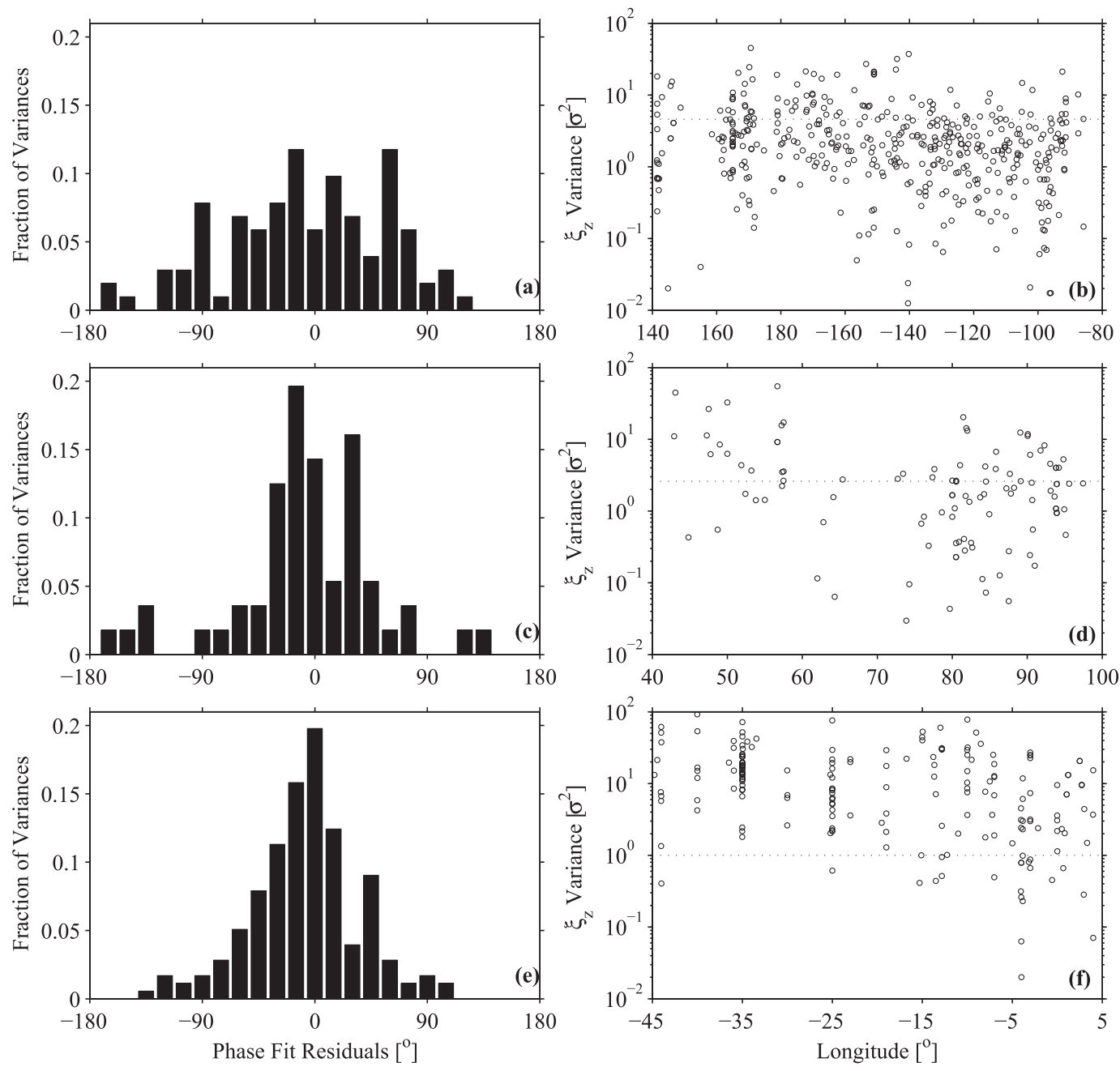

FIG. 10. Histograms of residuals of plane wave fits (Table 2) to phase estimates from vertical strain spectra at the pressures and vertical wavelengths used in Figs. 8 and 9 for the (a) Pacific Ocean using profiles with variance exceeding a 4.6 cutoff within $\pm 0.11^{\circ}$ of $\pm 1.4^{\circ}$ latitude, (c) Indian Ocean exceeding 2.6 variance within $\pm 0.17^{\circ}$ of $\pm 1.4^{\circ}$ latitude, and (e) Atlantic Ocean exceeding 1.0 variance within $\pm 0.6^{\circ}$ of $\pm 1.5^{\circ}$ latitude. Variance for the stations (o's) within those latitude ranges plotted vs longitude along with the three cutoff levels used (vertical dotted lines) in the (b) Pacific, (d) Indian, and (f) Atlantic Oceans.

in the western Pacific, although they classify it as broadband noise. Thus, the signature of the Rossby wave is present even in Johnson et al. (2002), but they did not have sufficient off-equatorial deep CTD casts to find its coherent pattern across the basin. Iridium Argo floats have remedied that situation, allowing new insights into basinwide, off-equatorial, Pacific, Rossby wave-like signatures in the present analysis.

Both Muench et al. (1994) in the Pacific and Johnson and Zhang (2003) in the Atlantic suggested that the observed EDJ signatures were about 1.5 times broader than their theoretical meridional scales. Our results agree with these two studies, extending that pattern to the Indian Ocean. Muench et al. (1994) suggest that the presence of high-frequency motion aliases the observed meridional scale. In a modeling study, Greatbatch et al. (2012) consider this widening, suggesting mixing of momentum along isopycnals as the cause. They find a widening by a factor of 1.5 over the linear theory for a realistic value of the diffusion coefficient.

Johnson and Zhang (2003) analyze vertical strain data and find the EDJs in the Atlantic to be primarily first meridional mode Rossby waves with a period of $5 \mathrm{yr}$, a zonal wavelength the order of the basin width at the equator, and downward phase propagation. Analyses of velocity data by Brandt et al. (2011) show results consistent with these findings. Our analysis confirms these results with a somewhat longer sampling period. In the power spectra computed in Johnson and Zhang (2003) there is also a broadband peak located at a vertical 
wavelength slightly shorter than the Rossby signature, although the Rossby wave peak is much more powerful. Eriksen (1982) recognizes the Kelvin wave component in vertical displacement profiles by phase relations at the equator, but a secondary peak in energy is seen around $\pm 1.5^{\circ}$ latitude at $36^{\circ} \mathrm{W}$, consistent with a first meridional mode Rossby wave.

In the Indian Ocean, a broad peak was seen by Ponte and Luyten (1990), with a range of 500-429 sm. The signal at 429 -sm vertical wavelength was classified as a Kelvin wave, which agrees with our analysis. The signal at $500 \mathrm{sm}$ is then likely the first meridional mode, equatorial, Rossby wave-like signature that we find. The peak at 660 -sm vertical wavelength seen by Dengler and Quadfasel (2002) was found to be a first meridional mode Rossby wave, consistent with our analysis. Of course, the temporal and zonal coverage afforded by the CTD profiles allows us to make relatively robust estimates of the period and, to a lesser extent, the zonal wavelength of this signature.

It has also been suggested (e.g., d'Orgeville et al. 2007; Bunge et al. 2008; Brandt et al. 2012) that the EDJs resemble basin modes (Cane and Moore 1981), which include equatorial Kelvin and long Rossby waves with zonal wavelengths equal to the basin width. The basin mode period is equivalent to the sum of the Kelvin and Rossby wave periods, so $4 / 3$ that of the Rossby wave alone. The zonal wavelengths on the order of the width of the basins found here in all three oceans, at least for the Rossby wave-like signals, are quite suggestive in this regard. Also in agreement with our findings, the meridional scale of these waves is broadened by mean zonal current structure, at least in the Atlantic (Claus et al. 2014), and to a greater extent by eddy viscosity (Greatbatch et al. 2012), both of which eliminate the formation of a midbasin caustic (Claus et al. 2014). However, there are some aspects of the observations that are less consistent with a basin mode. Our inability to detect a coherent, basinwide, equatorial Kelvin wave signal may be one discrepancy. Also, the observed variance of the off-equatorial strain at the Rossby wave peak vertical wavelength increases to the west in all three basins (Fig. 10), at odds with the signature of a simple modeled basin mode, where the Rossby wave signature dissipates with distance from the eastern boundary (Claus et al. 2014). The observed variance at the peak vertical wavelength variance at the equator (not shown) is fairly uniform with longitude in all three basins, where that simple modeled basin mode might exhibit variance decaying in amplitude from west to east.

The differences and similarities among the characteristics of the EDJ signatures in the three different oceans may help to narrow the possible range of plausible generation mechanisms for the EDJs. One modeling study, McCreary (1984) suggests that EDJs are superpositions of many long wavelength Kelvin and Rossby waves, but the most visible in the model are a Kelvin wave and a first meridional mode Rossby wave. The deep jets have also been theorized to be generated by unstable mixed Rossby-gravity waves (e.g., Hua et al. 2008; Ascani et al. 2010). If that theory holds, how might it explain the differences in amplitude in the Atlantic and the other two oceans? Another theory for EDJ generation includes large vertical-scale instability in western boundary currents (d'Orgeville et al. 2007). In support of this theory, the presence of stronger deep western boundary currents in the Atlantic than in the other two oceans might help explain the largeramplitude EDJ signals there. Also, the upward energy propagation observed may be consistent with a deep western boundary current source, since a deep energy source might imply upward energy propagation, away from that source (Brandt et al. 2011). Ascani et al. (2015) find that deep signatures of tropical instability waves in an idealized numerical Atlantic Ocean rectify into lowfrequency baroclinic Kelvin and Rossby waves (the EDJs) that form resonant basin modes. Their numerical EDJ characteristics are in broad agreement with our results. However, their EDJs weaken when realistic coastlines and seasonally varying winds are included in the model.

Acknowledgments. MKY was supported by the NOAA Hollings Scholar Program, and GCJ was supported by NOAA research. We thank all those who helped to collect, process, calibrate, and archive the shipboard CTD and Argo data. The Argo program is part of the Global Ocean Observing System. Argo data are collected and made freely available by the International Argo Program and the national programs that contribute to it (http://www.argo.ucsd.edu; http:// argo.jcommops.org). We also thank François Ascani, Eric Firing, William Kessler, Dennis Moore, LuAnne Thompson, and two anonymous reviewers for useful discussions and comments.

\section{REFERENCES}

Ascani, F., E. Firing, P. Dutrieux, J. P. McCreary, and A. Ishida, 2010: Deep equatorial ocean circulation induced by a forceddissipated Yanai beam. J. Phys. Oceanogr., 40, 1118-1142, doi:10.1175/2010JPO4356.1.

,,- J. P. McCreary, P. Brandt, and R. J. Greatbatch, 2015: The deep equatorial ocean circulation in wind-forced numerical solutions. J. Phys. Oceanogr., 45, 1709-1734, doi:10.1175/JPO-D-14-0171.1. 
Brandt, P., A. Funk, V. Hormann, M. Dengler, R. J. Greatbatch, and J. M. Toole, 2011: Interannual atmospheric variability forced by the deep equatorial Atlantic Ocean. Nature, 473, 497-500, doi:10.1038/nature10013.

— by the equatorial deep jets. J. Geophys. Res., 117, C12015, doi:10.1029/2012JC008118.

Bunge, L., C. Provost, B. L. Hua, and A. Kartavtseff, 2008: Variability at intermediate depths at the equator in the Atlantic Ocean in 2000-06: Annual cycle, equatorial deep jets, and intraseasonal meridional velocity fluctuations. J. Phys. Oceanogr., 38, 1794-1806, doi:10.1175/2008JPO3781.1.

Cane, M. A., and D. W. Moore, 1981: A note on low-frequency equatorial basin modes. J. Phys. Oceanogr., 11, 1578-1584, doi:10.1175/1520-0485(1981)011<1578:ANOLFE>2.0.CO;2.

Claus, M., R. J. Greatbatch, and P. Brandt, 2014: Influence of the barotropic mean flow on the width and the structure of the Atlantic equatorial deep jets. J. Phys. Oceanogr., 44, 24852497, doi:10.1175/JPO-D-14-0056.1.

Dengler, M., and D. Quadfasel, 2002: Equatorial deep jets and abyssal mixing in the Indian Ocean. J. Phys. Oceanogr., 32, 1165-1180, doi:10.1175/1520-0485(2002)032<1165: EDJAAM $>2.0 . \mathrm{CO} ; 2$.

d'Orgeville, M., B. L. Hua, and H. Sasaki, 2007: Equatorial deep jets triggered by a large vertical scale variability within the western boundary layer. J. Mar. Res., 65, 1-25, doi:10.1357/ 002224007780388720.

England, M. H., and Coauthors, 2014: Recent intensification of wind-driven circulation in the Pacific and the ongoing warming hiatus. Nat. Climate Change, 4, 222-227, doi:10.1038/ nclimate2106.

Eriksen, C. C., 1981: Deep currents and their interpretation as equatorial waves in the western Pacific Ocean. J. Phys. Oceanogr., 11, 48-70, doi:10.1175/1520-0485(1981)011<0048: DCATIA $>2.0 . \mathrm{CO} ; 2$.

_ 1982: Geostrophic equatorial deep jets. J. Mar. Res., 40,143157.

Firing, E., 1987: Deep zonal currents in the central equatorial Pacific. J. Mar. Res., 45, 791-812, doi:10.1357/002224087788327163.

Gouriou, Y., B. Bourlès, H. Mercier, and R. Chuchla, 1999: Deep jets in the equatorial Atlantic Ocean. J. Geophys. Res., 104, 21 217-21 226, doi:10.1029/1999JC900057.

- , and Coauthors, 2001: Deep circulation in the equatorial Atlantic Ocean. Geophys. Res. Lett., 28, 819-822, doi:10.1029/ 2000GL012326.

Greatbatch, R., P. Brandt, M. Claus, S.-H. Didwischus, and Y. Fu, 2012: On the width of the equatorial deep jets. J. Phys. Oceanogr., 42, 1729-1740, doi:10.1175/JPO-D-11-0238.1.

Hua, B. L., M. D'orgeville, M. D. Fruman, C. Menesguen, R. Schopp, P. Klein, and H. Sasaki, 2008: Destabilization of mixed Rossby gravity waves and the formation of equatorial zonal jets. J. Fluid Mech., 610, 311-341, doi:10.1017/ S0022112008002656.

Johnson, G. C., and D. Zhang, 2003: Structure of the Atlantic Ocean equatorial deep jets. J. Phys. Oceanogr., 33, 600-609, doi:10.1175/1520-0485(2003)033<0600:SOTAOE $>2.0$. CO;2.

— - E. Kunze, K. E. McTaggart, and D. W. Moore, 2002: Temporal and spatial structure of the equatorial deep jets in the Pacific Ocean. J. Phys. Oceanogr., 32, 3396-3407, doi:10.1175/ 1520-0485(2002)032<3396:TASSOT>2.0.CO;2.

Kessler, W. S., and J. P. McCreary, 1993: The annual winddriven Rossby wave in the subthermocline equatorial Pacific. J. Phys. Oceanogr., 23, 1192-1207, doi:10.1175/ 1520-0485(1993)023<1192:TAWDRW > 2.0.CO;2.

Leaman, K. D., and T. B. Sanford, 1975: Vertical energy propagation of inertial waves: A vector spectral analysis of velocity profiles. J. Geophys. Res., 80, 1975-1978, doi:10.1029/ JC080i015p01975.

Leetmaa, A., and P. F. Spain, 1981: Results from a velocity transect along the equator from 125 to $159^{\circ} \mathrm{W}$. J. Phys. Oceanogr., 11, 1030-1033, doi:10.1175/1520-0485(1981)011<1030: RFAVTA $>2.0 . \mathrm{CO} ; 2$.

Luyten, J. R., and J. C. Swallow, 1976: Equatorial undercurrents. Deep-Sea Res. Oceanogr. Abstr., 23, 999-1001, doi:10.1016/ 0011-7471(76)90830-5.

McCreary, J. P., Jr., 1984: Equatorial beams. J. Mar. Res., 42, 395430, doi:10.1357/002224084788502792.

Muench, J., E. Kunze, and E. Firing, 1994: The potential vorticity structure of equatorial deep jets. J. Phys. Oceanogr., 24, 418-428, doi:10.1175/1520-0485(1994)024<0418: TPVSOE $>2.0 . \mathrm{CO} ; 2$.

Ponte, R. M., and J. Luyten, 1989: Analysis and interpretation of deep equatorial currents in the central Pacific. J. Phys. Oceanogr., 19, 1025-1038, doi:10.1175/1520-0485(1989)019<1025: AAIODE $>2.0 . C O ; 2$.

$\longrightarrow$, and - 1990: Deep velocity measurements in the western equatorial Indian Ocean. J. Phys. Oceanogr., 20, 44-52, doi:10.1175/1520-0485(1990)020<0044:DVMITW>2.0.CO;2.

Ropelewski, C., and P. Jones, 1987: An extension of the TahitiDarwin Southern Oscillation index. Mon. Wea. Rev., 115, 2161-2165, doi:10.1175/1520-0493(1987)115<2161: AEOTTS $>2.0 . \mathrm{CO} ; 2$.

Schmid, C., B. Bourlès, and Y. Gouriou, 2005: Impact of the equatorial deep jets on estimates of zonal transports in the Atlantic. Deep-Sea Res. II, 52, 409-428, doi:10.1016/ j.dsr2.2004.12.008.

Torrence, C., and G. P. Compo, 1998: A practical guide to wavelet analysis. Bull. Amer. Meteor. Soc., 79, 61-78, doi:10.1175/ 1520-0477(1998)079<0061:APGTWA > 2.0.CO;2.

Weisberg, R., and A. Horigan, 1981: Low-frequency variability in the equatorial Atlantic. J. Phys. Oceanogr., 11, 913-920, doi:10.1175/1520-0485(1981)011<0913:LFVITE>2.0.CO;2. 